\title{
Cell-free cloning using multiply-primed rolling circle amplification with modified RNA primers
}

\author{
Hirokazu Takahashi ${ }^{1}$, Kimiko Yamamoto ${ }^{2}$, Toshio Ohtani ${ }^{1}$, and Shigeru Sugiyama ${ }^{1}$ \\ ${ }^{1}$ NanoBiotechnology Laboratory, Food Engineering Division, National Food Research Institute, National \\ Agriculture and Food Research Organization, Ibaraki, Japan and 'Insect Genome Laboratory, National Institute of \\ Agrobiological Sciences, Ibaraki, Japan
}

BioTechniques 47:609-615 (July 2009) doi 10.2144/000113155

Keywords: multiply-primed rolling circle amplification; random RNA primers; $\$ 29$ DNA polymerase; single molecule amplification; cell-free cloning

The predominant method for DNA cloning is by propagation in biological hosts, but this method has limitations because certain sequences are difficult to clone using any combination of available hosts or vectors. Recently, multiplyprimed rolling circle amplification (MPRCA) has been applied to overcome the problems of the DNA cloning via host cells. However, when MPRCA is used to amplify from minute quantities of DNA template, the products are mostly by-product DNA molecules generated by false priming and primer dimer formation. This study demonstrates that MPRCA using random RNA primers-instead of DNA primers-blocked the synthesis of by-products and succeeded in amplifying one copy of a circular DNA molecule more than 1012-fold to give microgram quantities of amplification product without using submicroliter reaction volumes. Furthermore, a ligation strategy was elaborated to circularize only the desired DNA sequence and eliminate undesired ligation-products. A combination of these methods was able to amplify and ligate a large construct without undesired DNA sequences and at microgram quantities within one day. Therefore, these methods have the possibility to improve DNA cloning techniques that have been restricted by the limitations of PCR methods or by the host cell.

\section{Introduction}

The most popular method for cloning foreign DNA is still via propagation in vectors-predominantly plasmids-using biological hosts such as Escherichia coli, followed by their isolation. However, these methods have limitations, because many sequences are difficult to clone using any combination of available hosts or vectors. For example, retroviral long-terminal repeats are not maintained stably in host cells (1), and large constructs, such as genetargeting vectors to produce knockout mice, frequently show low transformation efficiencies (2).

PCR is a common method used in the cell-free cloning of DNA molecules. However, PCR is not suitable to amplify highly repetitious or large sequences. For these reasons, a new cell-free enzymatic system for cloning difficult DNA sequences has long been desired. Recently, Hutchison and colleagues reported a novel cell-free cloning method using multiply-primed rolling circle amplification (MPRCA), which can amplify individual DNA molecules using submicroliter reaction volumes (3). This method provides one way to overcome the problems of foreign DNA cloning via host cells, because MPRCA, which is based on rolling circle amplification (RCA), has little limitation in terms of the amplification length or sequence (3-6). MPRCA has also been applied to the amplification for circular DNA directly from ligation reactions, including cDNA libraries $(5,7)$.

MPRCA was developed using bacteriophage $\phi 29$ DNA polymerase and protected random DNA primers as a method to amplify circular DNA molecules (8). Bacteriophage $\phi 29$ DNA polymerase has exceptional processing, high proofreading, and efficient strand displacement activities (9-11); thus, the amplified product is useful for many downstream analyses $(4,5,7,8,12-21)$. The crucial point of this method is the use of random DNA primers; thereby, this method can amplify the target
DNA without prior sequence information, such as in the amplification of a novel circular DNA virus $(14,15)$. However, the use of random DNA primers contributes to a critical problem with MPRCA. When MPRCA is used to amplify minute quantities of DNA template, downstream analyses are limited because most of the resulting molecules are by-products generated from undesired primer-directed DNA synthesis $(22,23)$. These by-products are also found in negative control samples without adding template DNA (22). Therefore, it is necessary to judge the success or failure of the amplification by other analyses such as restriction enzyme digestion, sequencing, or PCR amplification of specific regions $(3,18,24-26)$. Although optimized reaction conditions, including modified primers, have been reported to reduce the levels of by-products (22-24,27), these modified methods were almost completely ineffective in increasing the yield of desired DNA product via long reaction periods $(24,27)$. 
A

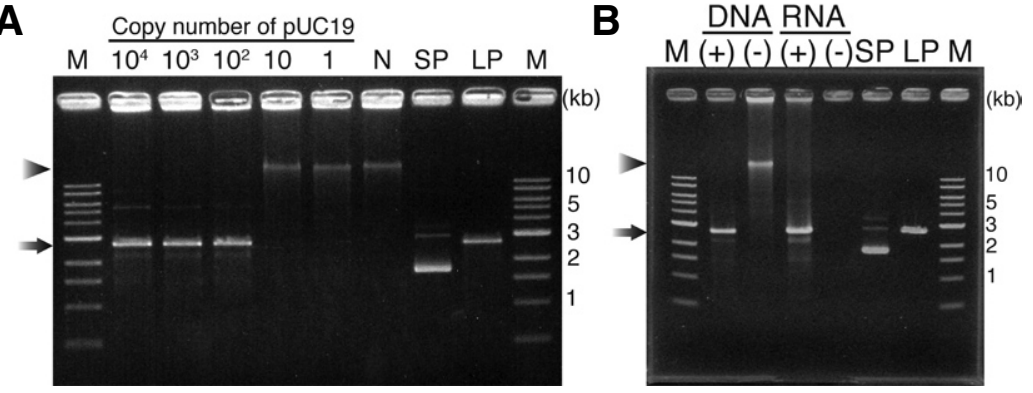

Figure 1. Gel analysis of by-product synthesis derived from the primers. (A) Template titration assays of MPRCA using DNA primer (6D2S; see Table 1). The amplification products were digested with $E c o R I$ and analyzed by agarose gel electrophoresis ( $2.5 \%$ of the original reaction product). The numbers above each lane indicate the input copy numbers of pUC19, calculated on the basis of dilution factors from a stock of known concentration (Takara Bio). M, 1-kb DNA ladder marker (New England Biolabs); N, no input DNA; SP, 100 ng supercoiled-form pUC19 (Takara Bio); LP, 100 ng linear-form pUC19 digested with EcoRI. Arrows indicate the 2.7-kb linear-form pUC19, and arrowheads indicate by-product from primers. (B) Agarose gel electrophoresis analyses of amplified products using DNA or RNA primers. Electrophoresis was performed after EcoRI digestion (2.5\% of the original reaction product). The type of primer [DNA (6D2S; see Table 1) or RNA (6RN; see Table 1)] used in the MPRCA is indicated at the top. M, 1-kb DNA ladder marker (New England Biolabs); SP, $100 \mathrm{ng}$ supercoiled-form pUC19 (Takara Bio); LP, 100 ng linear-form pUC19 digested; (+), input $10^{4}$ copies of pUC19; (-), no input DNA. Arrows indicate the 2.7-kb linear-form pUC19, and arrowheads indicate by-product from primers. These amplification reactions were repeated three times.

Here, we report that random RNA primers can block the synthesis of by-products derived from primers in the MPRCA reaction. Bacteriophage $\phi 29$ DNA polymerase can use RNA as a primer for DNA synthesis (28), but cannot use RNA as a template because $\phi 29$ DNA polymerase is a DNA-dependent DNA polymerase (29). We found that RNA-primed MPRCA blocked the synthesis of by-products derived from the primers and could amplify one molecule of circular DNA without using the submicroliter reaction volumes employed by Hutchison et al. (3). Because general ligation strategies circularize not only the desired DNA products but also undesired-products derived from self ligation (21), we also developed a ligation strategy to apply our amplification method in cell-free cloning of a ligated construct.

\section{Materials and methods}

Solutions, mixtures, plasticware, and DNA

To avoid contamination from the laboratory environment $(24,30)$, solutions supplied by the manufacturers were used as much as possible in this study. UltraPURE distilled water (dDW) and $1 \mathrm{M}$ Tris- $\mathrm{HCl}$ ( $\mathrm{pH}$ 7.5) were purchased from Invitrogen (Carlsbad, CA, USA), and 0.5 M EDTA was from NipponGene (Tokyo, Japan). Similarly, the plasticware used had already been sterilized by the supplier: aerosol resistant filter tips were purchased from Molecular BioProducts (San Diego, CA, USA), and Biopur microcentrifuge tubes and PCR-grade $0.2-\mathrm{mL}$ tubes were from Eppendorf(Hamburg, Germany). Purified
pUC19 was purchased from Takara Bio (Otsu, Shiga, Japan); $\lambda$ phage DNA was from TOYOBO (Osaka, Japan); and 1-kb DNA ladder marker was from New England BioLabs (Ipswich, MA, USA). Other solutions and reaction mixtures were prepared using a dedicated set of pipets (Finnpipette Focus; Thermo Fisher Scientific, Vantaa, Finland) in a dedicated clean bench after cleaning using RNase AWAY (Molecular Bioproducts, San Diego, CA, USA).

\section{Oligonucleotides}

Protected random DNA primers (6D2S, Table 1) were purchased from Hokkaido System Science (Sapporo, Japan). All RNA oligo-ribonucleotides, including modified RNA residues (Table 1), were purchased from Tsukuba Oligo Service (Tsukuba, Ibaraki, Japan). All random oligomers were dissolved with TE buffer $(10 \mathrm{mM}$ Tris- $\mathrm{HCl}$ $\mathrm{pH} 7.5,0.1 \mathrm{mM}$ EDTA) in the dedicated clean bench as described above.

\section{Control plasmid preparation}

The copy number of commercial pUC19 $(0.5 \mu \mathrm{g} / \mu \mathrm{L}$, Takara Bio) was calculated from its molecular weight. Control plasmid DNA solutions were prepared by serial dilution of pUC19 using TE buffer. The control solutions were prepared in a dedicated clean bench, though different than the one used to prepare MPRCA reagent to avoid the contamination to MPRCA reagents (30).

\section{MPRCA reaction}

The MPRCA reaction was performed based on the original procedure as described by Dean et al. (8). Two microliters of each
Table 1. Oligonucleotides Tested as Primers in This Study

\begin{tabular}{|c|c|}
\hline Primer name & Sequence \\
\hline $6 \mathrm{D} 2 \mathrm{~S}$ & 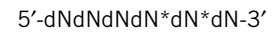 \\
\hline $6 \mathrm{RN}$ & $5^{\prime}-\mathrm{rNrNrNrNrNrN-}{ }^{\prime}$ \\
\hline $6 \mathrm{R} 5 \mathrm{~S}$ & $5^{\prime}-\mathrm{rN}{ }^{*} \mathrm{rN} * \mathrm{rN}{ }^{*} \mathrm{rN}{ }^{*} \mathrm{rN} * \mathrm{rN}-3^{\prime}$ \\
\hline $6 \mathrm{mN}$ & $5^{\prime}-\mathrm{mNmNmNmNmNmN-3^{ \prime }}$ \\
\hline $5 \mathrm{~m} 1 \mathrm{R}$ & 5'-mNmNmNmNmNrN-3' \\
\hline $4 \mathrm{~m} 2 \mathrm{R}$ & $5^{\prime}-\mathrm{mNmNmNmNrNrN-3^{ \prime }}$ \\
\hline \multicolumn{2}{|c|}{$\begin{array}{l}\text { dN: random DNA base } \\
\mathrm{rN} \text {, random RNA base } \\
\mathrm{mN} \text { : random 2'-O-methyl RNA base } \\
\text { *thiophosphate-linkage }\end{array}$} \\
\hline
\end{tabular}

control plasmid solution (see each figure legend) were added to $8 \mu \mathrm{L}$ annealing buffer, yielding a final concentration of 20 $\mu \mathrm{M}$ random hexamer (DNA or RNA; see Table 1), $30 \mathrm{mM}$ Tris- $\mathrm{HCl}$ (pH 7.5), 20 $\mathrm{mM} \mathrm{KCl}$, and $8 \mathrm{mM} \mathrm{MgCl}_{2}$. The plasmid was denatured for $1 \mathrm{~min}$ at $95^{\circ} \mathrm{C}$ and cooled slowly to $25^{\circ} \mathrm{C}$ over $30 \mathrm{~min}$. Reactions without target DNA contained an equivalent volume of $\mathrm{dDW}$. The $2 \times$ amplification premix $(10 \mu \mathrm{L})$ was added, yielding a final concentration of $35 \mathrm{mM}$ Tris- $\mathrm{HCl}(\mathrm{pH}$ 7.5), $50 \mathrm{mM} \mathrm{KCl}, 14 \mathrm{mM} \mathrm{MgCl} 2,10 \mathrm{mM}$ $\left(\mathrm{NH}_{4}\right)_{2} \mathrm{SO}_{4}, 4 \mathrm{mM}$ dithiothreitol (DTT), 40 U RepliPHI Phi29 DNA polymerase (Epicenter Biotechnologies, Madison, WI, USA), and $0.002 \mathrm{U}$ of inorganic pyrophosphatase (Roche Diagnostics, Penzberg, Germany). The reaction was performed for $16 \mathrm{~h}$ at $30^{\circ} \mathrm{C}$ followed by incubation for $10 \mathrm{~min}$ at $65^{\circ} \mathrm{C}$ to inactivate the enzyme. To reduce the high viscosity of the amplification products, they were transferred to $1.5-\mathrm{mL}$ centrifuge tubes and mixed with $180 \mu \mathrm{L}$ dDW by pipetting followed by vortex mixing.

\section{Restriction fragment analysis of MPRCA products}

Twenty-five microliters of diluted products (12.5\% of the original reaction product) were digested with 20 units of EcoRI (Takara Bio) for $1 \mathrm{~h}$ at $37^{\circ} \mathrm{C}$ in a $50-\mu \mathrm{L}$ volume. Then, $10 \mu \mathrm{L}$ of each digested sample $(2.5 \%$ of the original reaction product) were analyzed by electrophoresis using an agarose gel (1.0\%, Tris-AcetateEDTA buffer) and visualized by staining with ethidium bromide.

\section{Cell-free cloning}

Preparation of DNA fragments. Ten micrograms of $\lambda$ DNA were digested with $50 \mathrm{U}$ of $E c o$ RI for $1 \mathrm{~h}$ at $37^{\circ} \mathrm{C}$. After ethanol precipitation, this digested DNA was dephosphorylated using $40 \mathrm{U}$ calf intestinal alkaline phosphatase (CIAP) (Takara Bio) for $1 \mathrm{~h}$ at $37^{\circ} \mathrm{C}$. After phenol/ chloroform extraction to remove CIAP, the dephosphorylated DNA was digested 
A

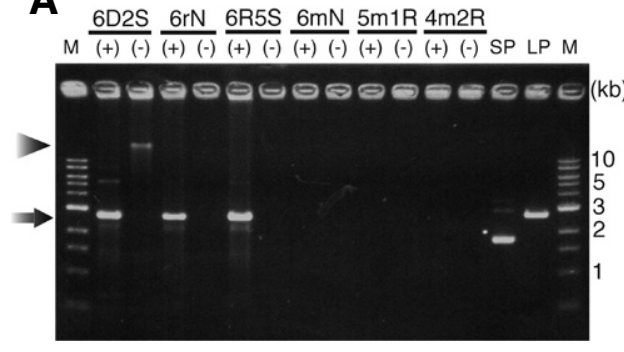

B

$$
\text { B }
$$

Copy number of pUC19

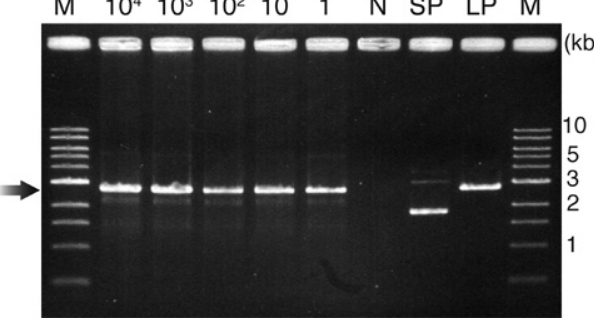

Figure 2. Gel analysis of MPRCA using modified RNA primers. (A) Agarose gel electrophoresis analysis of amplified DNA using several modified RNA primers. Electrophoresis was performed after EcoRI digestion (2.5\% of the original reaction product). The type of modified primers (see Table 1 ) used in MPRCA is indicated at the top. (+), input $10^{4}$ copies of pUC19; (-), no input DNA; M, 1-kb DNA ladder marker (New England Biolabs); SP, 100 ng supercoiled-form pUC19 (Takara Bio); LP, $100 \mathrm{ng}$ linear-form pUC19 digested with EcoRI. Arrows indicate the 2.7-kb linear-form pUC19, and arrowheads indicate by-product from primers. (B) Template titration assays of MPRCA using thiophosphate-linkage RNA primers (6R5S). Electrophoresis was performed after EcoRI digestion ( $2.5 \%$ of the original reaction product). The numbers above each lane indicate the input copy numbers of pUC19, which are the same as in Figure 1A. M, 1-kb DNA ladder marker (New England Biolabs); N, no input DNA; SP, 100 ng supercoiled-form pUC19 (Takara Bio); LP, 100 ng linear-form pUC19. Arrows indicate the $2.7-\mathrm{kb}$ linear-form pUC19. These amplification reactions were repeated three times.

with $40 \mathrm{U}$ of Bam HI (Takara Bio) for $1 \mathrm{~h}$ at $37^{\circ} \mathrm{C}$. Then, the $\lambda$ DNA fragment $(15.7$ $\mathrm{kb}$ ) was purified by agarose gel electrophoresis followed by extraction using a Wizard Gel and PCR purification system (Promega, Madison, WI, USA). Next, $5 \mu \mathrm{g}$ pUC19 were cleaved with $40 \mathrm{U}$ Bam HI for $1 \mathrm{~h}$ at $37^{\circ} \mathrm{C}$. The linear form of pUC19 was purified by agarose gel electrophoresis as described above. This purified linear pUC19 was dephosphorylated with CIAP for $1 \mathrm{~h}$ at $37^{\circ} \mathrm{C}$. After phenol/chloroform extraction, the dephosphorylated pUC19 was digested with $50 \mathrm{U} E c o \mathrm{RI}$ for $1 \mathrm{~h}$ at $37^{\circ} \mathrm{C}$. Then, the $B a m \mathrm{HI} / E c o \mathrm{RI}$ doubledigested pUC19 was purified by agarose gel electrophoresis as described above. The concentration of the purified DNA was determined using a Quant-iT dsDNA HS assay kit (Invitrogen) and Qubit fluorometer (Invitrogen).

Ligation reaction and exonuclease treatment. Ligation reactions were performed in a $10-\mu \mathrm{L}$ volume for $30 \mathrm{~min}$ at $16^{\circ} \mathrm{C}$ at a concentration of $1 \times 10^{8}$ copies $/ \mu \mathrm{L}$ $\lambda$ DNA fragment and $1 \times 10^{8}$ copies $/ \mu \mathrm{L}$ pUC19 in T4 DNA ligase buffer with 200 U T4 DNA ligase (New England Biolabs) followed by incubation for $10 \mathrm{~min}$ at $65^{\circ} \mathrm{C}$ to inactivate the enzyme. The reaction without T4 DNA ligase contained an equivalent volume of $\mathrm{dDW}$. The ligated samples were diluted with $90 \mu \mathrm{L}$ dDW. Exonucleases were used to degrade linear but not circularized DNA to avoiding undesired DNA amplification. Two microliters of the diluted samples were treated with 10 U RecBCD exonuclease (Epicenter Biotechnologies) and $10 \mathrm{U}$ exonuclease I (New England Biolabs) in a $20-\mu \mathrm{L}$ volume of RecBCD buffer with $1 \mathrm{mM}$ ATP for 4 $\mathrm{h}$ at $37^{\circ} \mathrm{C}$ followed by incubation for 20 $\min$ at $80^{\circ} \mathrm{C}$ to inactivate the enzymes. The reactions without exonuclease contained

\section{Give your marketing programs a LIFT!}

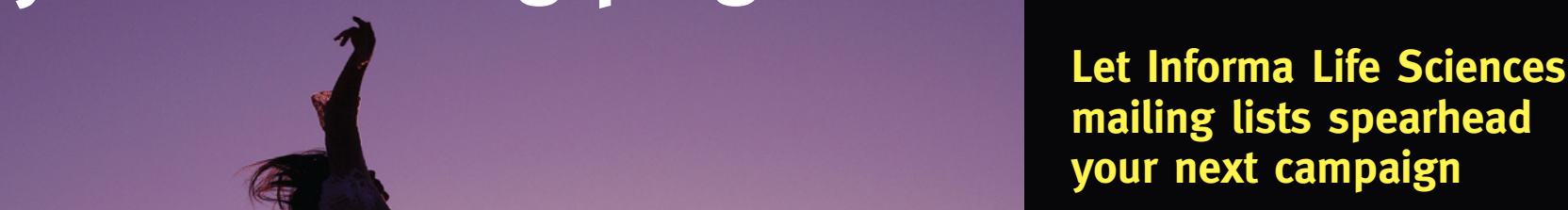

Let Informa Life Sciences mailing lists spearhead your next campaign

If you are looking to maximize your marketing efforts to professionals who work in all aspects of pharma and biotech, you need a resource that gives you access to your best responders - and that's Informa Life Science mailing lists.

Cultivated from our targeted subscriber base of life science professionals, our lists give you the best chance of delivering your brand and offer to this market.

Let us locate the right decision-makers in your market segment:

\section{- Over 125,000 life science professionals}

- $\$ 25$ billion in purchasing power

- Segmentation including function, title and laboratory technique - worldwide reach 
A

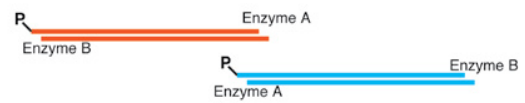

B

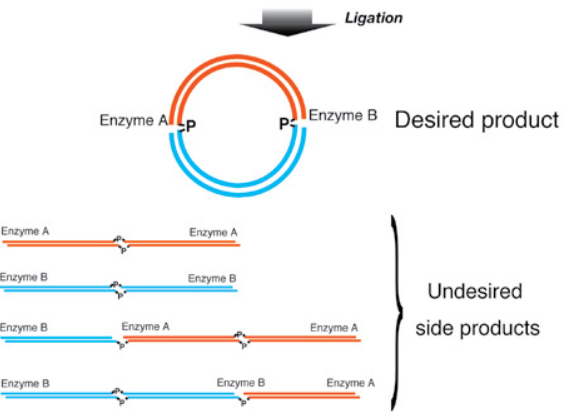

C

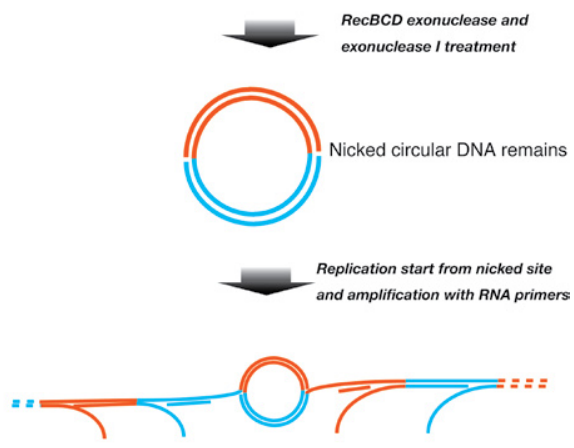

E

$\begin{array}{llllll}M & 1 & 2 & 3 & 4 & M\end{array}$

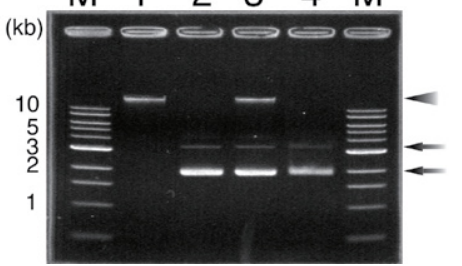

Figure 3. Schematic representation of the strategy for cell-free cloning using RNA-primed MPRCA. (A) The DNA fragments (red and blue lines), which were phosphorylated at the opposing restriction enzyme sites, were ligated using T4 DNA ligase. (B) In this case, the desired DNA molecule is in the open circular form, where one strand is completely circularized and the other strand has two nicked sites. Undesired ligation products with self-ligated sequences are in linear. (C) Ligated samples are treated using exonucleases to remove undesired linear DNA. (D) Finally, only the remaining circular DNA is replicated from the nicked sites using $3^{\prime}$ termini as first primers, and amplified with thiophosphate-linkage random RNA primers (6R5S). (E) Exonuclease-mediated removal of linear DNA from the mixture of linear and circular DNA molecules. Lane 1, $100 \mathrm{ng} \lambda \mathrm{DNA}$ (linear); Lane 2, $100 \mathrm{ng}$ pUC19 (circular); Lane 3, mixture of $100 \mathrm{ng} \lambda$ DNA and $100 \mathrm{ng}$ pUC19 before exonuclease treatment; Lane 4, mixture of $\lambda$ DNA fragment and supercoiled pUC19 after exonuclease treatment; M, 1-kb DNA ladder (New England Biolabs). Arrows indicate the circular forms of pUC19 and arrowhead indicates $\lambda$ DNA.

an equivalent volume of dDW. To verify the exonuclease treatment, a mixture of $\lambda$ DNA and pUC19 was used as a control for removing linear DNA while leaving circular DNA. The exonuclease-treated samples were diluted with $180 \mu \mathrm{L} \mathrm{dDW}$.

Amplification of the desired products. Two microliters of the diluted exonuclease-treated samples were used for the amplification reaction. The amplifications were performed in a $20-\mu \mathrm{L}$ volume containing $10 \mu \mathrm{M} 6 \mathrm{R} 5 \mathrm{~S}$ primer, $35 \mathrm{mM}$ Tris- $\mathrm{HCl}(\mathrm{pH}$ 7.5), $50 \mathrm{mM} \mathrm{KCl}, 14 \mathrm{mM}$ $\mathrm{MgCl}_{2}, 10 \mathrm{mM}\left(\mathrm{NH}_{4}\right)_{2} \mathrm{SO}_{4}, 4 \mathrm{mM}$ DTT,
40 U RepliPHI Phi29 DNA polymerase, and $0.002 \mathrm{U}$ inorganic pyrophosphatase for $16 \mathrm{~h}$ at $30^{\circ} \mathrm{C}$ followed by incubation for 10 min at $65^{\circ} \mathrm{C}$ to inactivate the enzyme. The reactions without target DNA contained equivalent volumes of $\mathrm{dDW}$. To reduce the high viscosity of the amplification products, they were diluted with $180 \mu \mathrm{L}$ $\mathrm{dDW}$ and mixed as described above.

Restriction fragment analyses of amplification products from the ligated construct. Twenty-five microliters of diluted amplification product from cell-free cloning were digested with $20 \mathrm{U} E c o \mathrm{RI}$,
$B a m \mathrm{HI}$ and EcoRI (double-digested), or $E c o$ RV (Takara Bio) for $3 \mathrm{~h}$ at $37^{\circ} \mathrm{C}$ in a total volume of $50 \mu \mathrm{L}$. The digested samples were desalted using Microspin S400 HR columns (GE Healthcare, Little Chalfont, UK). Then, a $20-\mu \mathrm{L}$ aliquot of each desalted sample ( $5 \%$ of the original reaction product) was analyzed by electrophoresis using an agarose gel $(0.8 \%$, Tris-acetate-EDTA buffer) and visualized by staining with ethidium bromide.

\section{Results}

Use of a random RNA primer

In principle, only the desired DNA sequence is amplified by MPRCA without by-products-even if the reaction is started from minute quantities of template DNA-because MPRCA is based on rolling circle replication or amplification $(11,29,31,32)$. However, with decreasing copy numbers of the template DNA sequences in the reaction, by-products can emerge. In the case where the template DNA is a plasmid, by-products can be detected easily using restriction endonuclease cleavage at the multiple-cloning site (3) because the by-products-especially those derived from random DNA primers (6D2S, Table 1)-generally have no predictable restriction endonuclease sites. Consistent with this, we found that nearly all the by-products migrated at $>20 \mathrm{kbp}$ in agarose gel electrophoresis (Figure 1A). By contrast, the MPRCA product from a circular template is a hyperbranched, multiple tandem concatemer and mostly double-stranded DNA (dsDNA) $(5,8)$. Thus, EcoRI digestion of the amplification products from $\mathrm{pUC} 19$ produced an obvious band corresponding to the linear form of pUC19 (about $2.7 \mathrm{~kb}$, Figure 1A). To block by-product synthesis derived from self-annealed primers, random RNA primers (6RN, Table 1) were employed in the MPRCA reaction. Bacteriophage $\phi 29$ DNA polymerase can use RNA as a primer for DNA synthesis (28), but it cannot use RNA as a template (29). There was little or no difference in the amplification products between the 6D2S and 6RN primers when the template was added to the reaction (Figure 1B). On the other hand, by-products were almost completely absent when 6RN primers were used in the absence of DNA template (Figure 1B).

Effect of modification of the RNA primer in the MPRCA reaction Because RNA is susceptible to degradation by RNases and the RNase activity of $\$ 29$ DNA polymerase $(33,34)$, it was considered necessary to modify the RNA primer using 2'-O-methyl RNA residues or 
A
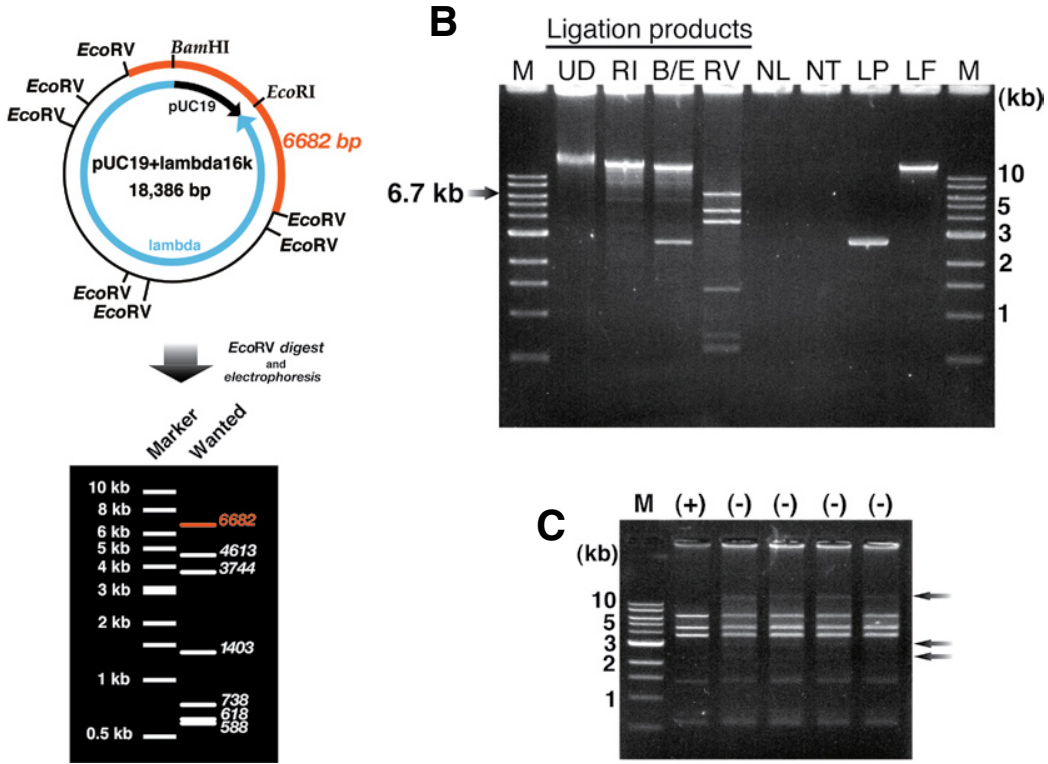

Figure 4. Cell-free cloning using RNA-primed MPRCA. (A) Restriction fragment map of the desired DNA in this experiment; $\lambda$ DNA BamHI/EcoRI fragment (15.7 kb, blue arrow) ligated into pUC19

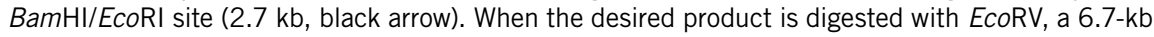
fragment (red color) should appear. (B) Verification of the amplification products by restriction fragment-length analyses. Electrophoresis was performed after restriction enzyme digestion $(5 \%$ of the original reaction product). UD, undigested amplification product; RI, EcoRI digested amplification product; $\mathrm{B} / \mathrm{E}, \mathrm{BamHI}$ and EcoRI double-digested amplification product; RV, EcoRV-digested amplification product; NL, not ligated; NT, no input DNA; LP, 100 ng linear-form pUC19; LF, 100 ng $\lambda$ 15.7-kb fragment; M, 1-kb DNA ladder (New England Biolabs). Arrow indicates the 6.7-kb $E c o R V$ fragment from the desired DNA molecule. These amplification reactions were repeated three times. (C) Verification of the amplification products with or without exonuclease treatment was by restriction fragment-length analyses. Electrophoresis was performed after EcoRV digestion (5\% of the original reaction product). (+), with exonuclease treatment; (-), without exonuclease treatment; M, 1-kb DNA ladder marker (New England Biolabs). Arrows indicate the undesired DNA molecule.

thiophosphate linkages for greater stability during the long reaction period. However, these modifications may have unexpected influences on DNA synthesis in MPRCA; therefore, we next examined RNA-primed MPRCA reactions using RNA primers with several types of modification (Table 1). It was found that DNA synthesis was inhibited when using 2'-O-methyl RNA residues $(6 \mathrm{mN}, 5 \mathrm{~m} 1 \mathrm{R}$, and $4 \mathrm{~m} 2 \mathrm{R}$; Table 1 and Figure 2A). On the other hand, when thiophosphate-linkage RNA primers (6R5S, Table 1) were used, no inhibitory influences on DNA synthesis were observed and by-products were eliminated (Figure 2A). Moreover, EcoRI cleavage of the amplification products from one copy of pUC19 ( 3 attograms) displayed a clear pUC19 linear-form band without the need to reduce the reaction volume (Figure 2B) and yielded about $4 \mu \mathrm{g}$ amplified product in a $20-\mu \mathrm{L}$ reaction volume, which was estimated by comparison with the control DNA (pUC19). The results show that MPRCA using thiophosphate-linkage RNA primers (6R5S) can amplify a single copy of a circular DNA molecule more than $10^{12}$-fold to give microgram quantities of amplification product without by-product. Importantly, this does not require the use of submicroliter reaction volumes, which were used in the method of Hutchison et al. (3).

Amplification of a ligated large construct Next, it was tested whether MPRCA using 6R5S primers could amplify large, ligated constructs. However, general ligation strategies circularize not only the desired product but also undesired self-ligated products. Thus, we needed to develop a strategy to circularize only the desired DNA constructs and to eliminate the undesired ligation products (Figure 3). To prevent circularization of the undesired ligation products, each DNA fragment is dephosphorylated at the opposite restriction enzyme site (Figure $3 \mathrm{~A})$. When these DNA fragments are incubated with T4 DNA ligase, the desired form (one strand completely circularized and the other strand with two nicked sites; Figure 3B). In contrast, undesired ligation products that have self-ligated would be linear (Figure 3B); however, these linear ligation products often have nicks that result from the ligation of three or more fragments (Figure 3B). These nicks could serve as starting sites for DNA synthesis by $\$ 29$ DNA polymerase, producing ligated product will be in the open circular undesired DNA amplification; therefore, these linear DNA molecules were eliminated by exonuclease treatment while leaving circular DNA molecules (Figure $3 C)$. Finally, circular DNA is replicated from the nicked sites using the $3^{\prime}$ termini of nicks as first primers and amplified using MPRCA with 6R5S primers (Figure 3D). To measure the efficiency of the removal of linear DNA by exonuclease treatment, a control DNA mixture including linear $\lambda$ DNA and circular pUC19 DNA was used (Figure 3E; Lanes 1, 2, and 3). We found that exonuclease treatment eliminated almost all of the linear $\lambda$ DNA while leaving the circular pUC19 DNA (Figure 3E; Lane 4).

As a model experiment, we ligated a Bam HI/EcoRI fragment of $\lambda$ DNA (15.7 $\mathrm{kbp}$ ) inserted into pUC19, and used the ligation products as an amplification template. The amplified products of cell-free cloning were checked for the amplification of the desired DNA sequence by restriction enzyme fragment-length analyses using agarose gel electrophoresis (Figure 4A). The EcoRV fragment $(6.7 \mathrm{~kb})$ that would be expected to be generated from the desired target DNA was detected in the ligated sample but not in an unligated sample or the negative controls (Figure 4B). Furthermore, by comparison with the amount of control DNA ( $\lambda 15.6 \mathrm{~kb})$, the yield of amplification product was about $4 \mu \mathrm{g}$ in a $20-\mu \mathrm{L}$ reaction volume run for 1 day. This yield should be enough to perform most downstream applications. In contrast, undesired DNA fragments were detected in the amplification reaction performed without exonuclease treatment (Figure 4C). Therefore, exonuclease treatment was an essential step for cell-free cloning to avoid undesired DNA amplification. The results demonstrate that the present ligation procedures combined with MPRCA using RNA primers could be suitable for cell-free cloning because the method suppresses undesired DNA amplification.

\section{Discussion}

Until now, RNA primers have not been favored for use in DNA amplification methods. In particular, the use of DNA primers in PCR has been an essential requirement for the following reasons: (i) the synthetic efficiency of RNA oligonucleotides is much lower than that of DNA oligonucleotides, which results in a high synthesis price for the RNA oligonucleotides compared with those for DNA oligonucleotides; (ii) Taq polymerase, the most popular polymerase for PCR, shows low efficiency when using RNA as a primer (35); and (iii) most importantly, 
for DNA synthesis of the antisense strand, the primer region is indispensable for PCR amplification so that primers can anneal to newly synthesized templates $(35,36)$.

None of these limitations precludes the use of RNA in MPRCA. This study demonstrates by-product-free DNA amplification from one copy of a circular DNA template using an RNA primer (6R5S; Figure 2A). Moreover, MPRCA using 6R5S primer did not require submicroliter reaction volumes to amplify one copy of circular DNA (Figure 2B); therefore, MPRCA using 6R5S primer could be performed in standard reaction volumes and the sensitivity of the 6R5S primer is higher than that of a DNA primer (6D2S) in the MPRCA reaction. On the other hand, the specificity of the 6R5S primer would be expected to be equal to that of the $6 \mathrm{D} 2 \mathrm{~S}$ primer because both primers have random sequences. These findings suggest that the versatility of the 6R5S primer could be comparable to that of the 6D2S primer in the MPRCA reaction.

After the submission of this manuscript, we became aware of a patent describing the use of RNA primers to suppress nonspecific RCA background (37). However, there are two differences between the patent and the present paper. First, the patent has been developed for the detection of single-strand circular probes such as a padlock-probe $(11,38)$; thus, the patent requires RNA primers with long, specific sequences to detect a specific probe. In contrast, the present study used random RNA primers with short sequences to expand the versatility of MPRCA. Second, differences in the length of the RNA primers affect the reaction temperatures used in these two methods. Because accurate annealing of a long, specific RNA primer requires a high temperature, the patented method needs Bst DNA polymerase (large fragment), which works optimally at $65^{\circ} \mathrm{C}$. In contrast, random hexamers are unsuitable for reactions at $65^{\circ} \mathrm{C}$, and the $\phi 29 \mathrm{DNA}$ polymerase-directed reaction is performed at $30^{\circ} \mathrm{C}$. In addition, the fidelity of $B s t$ DNA polymerase is lower than that of $\phi 29$ DNA polymerase, because Bst DNA polymerase lacks $3^{\prime}-5^{\prime}$ exonuclease activity (39). Thus, these differences indicate that combined use of specific RNA primers and Bst DNA polymerase is not suitable for the MPRCA reaction, especially not for cell-free cloning.
In this study, it was demonstrated that cell-free cloning of a ligated, large construct is one of the potential applications of RNA-primed MPRCA. This amplification method can amplify minute quantities of circular DNA: even if only a few copies of ligated circular products are available (due to low ligation efficiency, for example), this method could allow amplification of the desired DNA sequence. Furthermore, provided the number of DNA fragments is the same as the number of kinds of restriction enzyme used, this ligation strategy does not have any limit on the number of DNA fragments that can be used. The amplified DNA product may be suitable to use for the transformation of mammalian cells because the product DNA does not include endotoxins of bacterial origin. Recently, gene-synthesis technology with the elimination of sequence errors-as carried out using oligonucleotides, thermostable ligase, plural exonucleases, and endonuclease $\mathrm{V}$-has been reported (40). Although this method may provide great advantages in the chemical synthesis of genes, it has limitations originating from the use of PCR. MPRCA combined with RNA primers may overcome this

\section{BioTechniques \\ The International Journal of Life Science Methods}
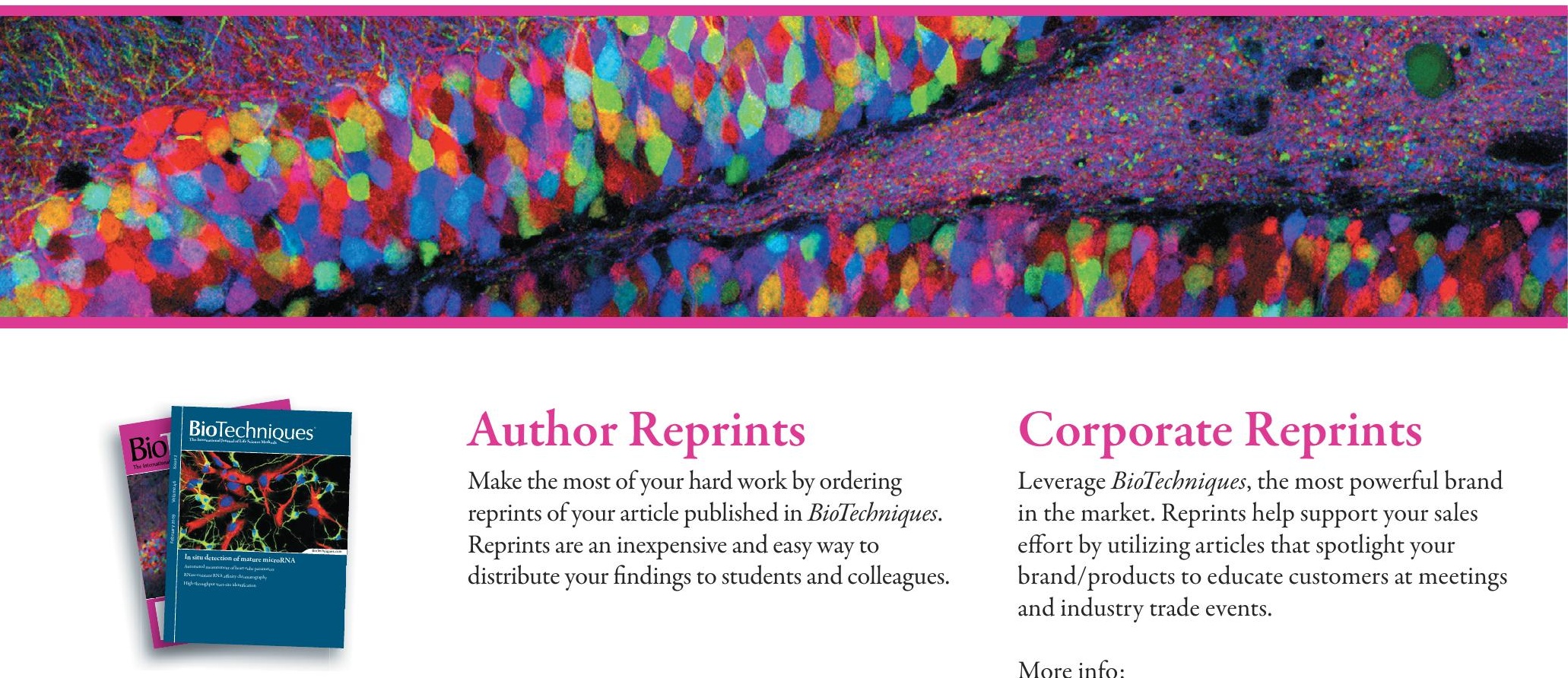

\section{Author Reprints}

Make the most of your hard work by ordering reprints of your article published in BioTechniques.

Reprints are an inexpensive and easy way to

distribute your findings to students and colleagues.

\section{Corporate Reprints}

Leverage BioTechniques, the most powerful brand in the market. Reprints help support your sales effort by utilizing articles that spotlight your brand/products to educate customers at meetings and industry trade events.

More info:

www.BioTechniques.com/reprints 
limitation because the desired product of the method is in a covalently closed circular duplex form.

Circular DNA amplification by MPRCA using RNA primers could greatly improve DNA cloning techniques that are restricted by the limitations of PCR (encountered with highly repetitious or large sequences) or by the host cell being used (encountered with unstable or toxic sequences in host cells) $(3,5,6)$.

\section{Acknowledgments}

The authors thank Jun'ichi Wakayama, Kazumi Tsukamoto, Toshiro Kobori, Yukio Magariyama, and members of the Nanobiotechnology group for helpful discussions; and Atsuko Matsumoto, Hiroko Kanahara, and Kanae Tsukada for excellent technical assistance. This work was supported in part by the Bio-Oriented Technology Research Advancement Institution.

The authors declare no competing interests.

\section{References}

1. Mager, D.L. and P.S. Henthorn. 1984. Identification of a retrovirus-like repetitive element in human DNA. Proc. Natl. Acad. Sci. USA 81:75107514 .

2. Inoue, H., H. Nojima, and H. Okayama. 1990. High efficiency transformation of Escherichia coli with plasmids. Gene 96:23-28.

3. Hutchison, C.A., 3rd, H.O. Smith, C. Pfannkoch, and J.C. Venter. 2005. Cell-free cloning using phi29 DNA polymerase. Proc. Natl. Acad. Sci. USA 102:17332-17336.

4. Berr, A. and I. Schubert. 2006. Direct labelling of BAC-DNA by rolling-circle amplification. Plant J. 45:857-862.

5. Christ, D., K. Famm, and G. Winter. 2006 Tapping diversity lost in transformations-in vitro amplification of ligation reactions. Nucleic Acids Res. 34:e108.

6. Osborne, R.J. and C.A. Thornton. 2008. Cell-free cloning of highly expanded CTG repeats by amplification of dimerized expanded repeats. Nucleic Acids Res. 36:e24.

7. Fullwood, M.J., J.J. Tan, P.W. Ng, K.P. Chiu, J. Liu, C.L. Wei, and Y. Ruan. 2008. The use of multiple displacement amplification to amplify complex DNA libraries. Nucleic Acids Res. 36:e32.

8. Dean, F.B., J.R. Nelson, T.L. Giesler, and R.S. Lasken. 2001. Rapid amplification of plasmid and phage DNA using Phi 29 DNA polymerase and multiply-primed rolling circle amplification. Genome Res. 11:1095-1099.

9. Blanco, L., A. Bernad, J.M. Lazaro, G. Martin, C. Garmendia, and M. Salas. 1989. Highly efficient DNA synthesis by the phage phi 29 DNA polymerase. Symmetrical mode of DNA replication. J. Biol. Chem. 264:8935-8940.

10. Esteban, J.A., M. Salas, and L. Blanco. 1993. Fidelity of phi 29 DNA polymerase. Comparison between protein-primed initiation and DNA polymerization. J. Biol. Chem. 268:2719-2726.
11. Lizardi, P.M., X. Huang, Z. Zhu, P. Bray-Ward, D.C. Thomas, and D.C. Ward. 1998. Mutation detection and single-molecule counting using isothermal rolling-circle amplification. Nat. Genet. 19:225-232.

12.Nelson, J.R., Y.C. Cai, T.L. Giesler, J.W. Farchaus, S.T. Sundaram, M. Ortiz-Rivera, L.P. Hosta, P.L. Hewitt, et al. 2002. TempliPhi, phi29 DNA polymerase based rolling circle amplification of templates for DNA sequencing. BioTechniques 32:S44-S47.

13. Wu, H.C., J. Shieh, D.J. Wright, and A. Azarani. 2003. DNA sequencing using rolling circle amplification and precision glass syringes in a high-throughput liquid handling system. BioTechniques 34:204-207.

14. Inoue-Nagata, A.K., L.C. Albuquerque, W.B. Rocha, and T. Nagata. 2004. A simple method for cloning the complete begomovirus genome using the bacteriophage phi29 DNA polymerase. J. Virol. Methods 116:209-211.

15. Rector, A., R. Tachezy, and M. Van Ranst. 2004. A sequence-independent strategy for detection and cloning of circular DNA virus genomes by using multiply primed rolling-circle amplification. J. Virol. 78:4993-4998.

16. Wang, G., E. Maher, C. Brennan, L. Chin, C. Leo, M. Kaur, P. Zhu, M. Rook, et al. 2004. DNA amplification method tolerant to sample degradation. Genome Res. 14:23572366.

17. Panelli, S., G. Damiani, L. Espen, and V. Sgaramella. 2005. Ligation overcomes terminal underrepresentation in multiple displacement amplification of linear DNA. BioTechniques 39:174-180.

18. Brukner, I., D. Labuda, and M. Krajinovic. 2006. Phi29-based amplification of small genomes. Anal. Biochem. 354:154-156.

19. Jakov, M.B. and P.D. Kassner. 2007. Multiple displacement amplification products are compatible with recombination-based cloning. BioTechniques 42:706, 708

20. Roohi, J., M. Cammer, C. Montagna, and E. Hatchwell. 2008. An improved method for generating BAC DNA suitable for FISH. Cytogenet. Genome Res. 121:7-9.

21. Shoaib, M., S. Baconnais, U. Mechold, E. Lecam, M. Lipinski, and V. Ogryzko. 2008. Multiple displacement amplification for complex mixtures of DNA fragments. BMC Genomics 9:415.

22.Lage, J.M., J.H. Leamon, T. Pejovic, S. Hamann, M. Lacey, D. Dillon, R. Segraves, B. Vossbrinck, et al. 2003. Whole genome analysis of genetic alterations in small DNA samples using hyperbranched strand displacement amplification and array-CGH. Genome Res. 13:294307.

23. Brukner, I., B. Paquin, M. Belouchi, D. Labuda, and M. Krajinovic. 2005. Self-priming arrest by modified random oligonucleotides facilitates the quality control of whole genome amplification. Anal. Biochem. 339:345-347.

24.Zhang, K., A.C. Martiny, N.B. Reppas, K.W. Barry, J. Malek, S.W. Chisholm, and G.M. Church. 2006. Sequencing genomes from single cells by polymerase cloning. Nat. Biotechnol. 24:680-686.

25. Kvist, T., B.K. Ahring, R.S. Lasken, and P. Westermann. 2007. Specific singlecell isolation and genomic amplification of uncultured microorganisms. Appl. Microbiol. Biotechnol. 74:926-935.

26. Holbrook, J.F., D. Stabley, and K. Sol-Church. 2005. Exploring whole genome amplification as a DNA recovery tool for molecular genetic studies. J. Biomol. Tech. 16:125-133.

27. Yokouchi, H., Y. Fukuoka, D. Mukoyama, R. Calugay, H. Takeyama, and T. Matsunaga. 2006. Whole-metagenome amplification of a microbial community associated with scleractinian coral by multiple displacement amplification using phi29 polymerase. Environ. Microbiol. 8:11551163.

28. Jonstrup, S.P., J. Koch, and J. Kjems. 2006. A microRNA detection system based on padlock probes and rolling circle amplification. RNA 12:1747-1752.

29. Kornberg, A. and T. Baker. 1992 DNA Replication. W.H. Freeeman and Co. San Francisco, CA.

30. Burkardt, H.J. 2000. Standardization and quality control of PCR analyses. Clin. Chem. Lab. Med. 38:87-91.

31. Fire, A. and S.Q. Xu. 1995. Rolling replication of short DNA circles. Proc. Natl. Acad. Sci. USA 92:4641-4645

32. Liu, D., S.L. Daubendiek, M.A. Zillman, K. Ryan, and E.T. Kool. 1996. Rolling circle DNA synthesis: small circular oligonucleotides as efficient templates for DNA polymerases. J Am. Chem. Soc. 118:1587-1594.

33. Bonnin, A., J.M. Lazaro, L. Blanco, and M. Salas. 1999. A single tyrosine prevents insertion of ribonucleotides in the eukaryotictype phi29 DNA polymerase. J. Mol. Biol. 290:241-251.

34. Lagunavicius, A., Z. Kiveryte, V. Zimbaite-Ruskuliene, T. Radzvilavicius, and A. Janulaitis. 2008. Duality of polynucleotide substrates for Phi29 DNA polymerase: $3^{\prime} \rightarrow 5^{\prime}$ RNase activity of the enzyme. RNA 29:503 513.

35. Shibata, H., T. Tahira, and K. Hayashi. 1995. RNA-primed PCR. Genome Res. 5:400403.

36. Stump, M.D., J.L. Cherry, and R.B. Weiss. 1999. The use of modified primers to eliminate cycle sequencing artifacts. Nucleic Acids Res. 27:4642-4648

37. Knott, T., C. Smith, J. Pickering, and T. Schwarz, inventors. Amersham Pharmacia Bioteck UK Limited. 2002. Suppression of non-specific nucleic acid amplification. Patent no. WO 02/057487 A2.

38. Nilsson, M., H. Malmgren, M. Samiotaki, M. Kwiatkowski, B.P. Chowdhary, and U. Landegren. 1994. Padlock probes: circularizing oligonucleotides for localized DNA detection. Science 265:2085-2088.

39. Aliotta, J.M., J.J. Pelletier, J.L. Ware, L.S Moran, J.S. Benner, and H. Kong. 1996. Thermostable Bst DNA polymerase I lacks a $3^{\prime} \rightarrow 5^{\prime}$ proofreading exonuclease activity. Genet. Anal. 12:185-195.

40. Bang, D. and G.M. Church. 2008. Gene synthesis by circular assembly amplification. Nat. Methods 5:37-39.

Received 6 October 2008; accepted 27 March 2009.

Address correspondence to Shigeru Sugiyama, Nano-Biotechnology Laboratory, Food Engineering Division, National Food Research Institute, National Agriculture and Food Research Organization, 2-1-12 Kannondai, Tsukuba, Ibaraki 3058642, Japan. e-mail: ssugi@affrc.go.jp 